\title{
Overall survival differences between patients with inflammatory and noninflammatory breast cancer presenting with distant metastasis at diagnosis
}

\author{
Tamer M. Fouad ${ }^{1,7,8}$, Takahiro Kogawa1,7, Diane D. Liu², Yu Shen², Hiroko Masuda ${ }^{1,7}$, \\ Randa El-Zein ${ }^{3,7}$, Wendy A. Woodward ${ }^{4,7}$, Mariana Chavez-MacGregor ${ }^{1,7}$, Ricardo H. \\ Alvarez $^{1,7}$, Banu Arun ${ }^{1}$, Anthony Lucci ${ }^{5,7}$, Savitri Krishnamurthy ${ }^{6,7}$, Gildy Babiera ${ }^{5}$, \\ Thomas A. Buchholz ${ }^{4}$, Vicente Valero ${ }^{1,7}$, and Naoto T. Ueno ${ }^{1,7}$ \\ Naoto T. Ueno: nueno@mdanderson.org \\ 1Department of Breast Medical Oncology, The University of Texas MD Anderson Cancer Center, \\ Houston, TX, USA \\ ${ }^{2}$ Department of Biostatistics, The University of Texas MD Anderson Cancer Center, Houston, TX, \\ USA \\ ${ }^{3}$ Department of Epidemiology, The University of Texas MD Anderson Cancer Center, Houston, \\ TX, USA \\ ${ }^{4}$ Department of Radiation Oncology, The University of Texas MD Anderson Cancer Center, \\ Houston, TX, USA \\ ${ }^{5}$ Department of Surgical Oncology, The University of Texas MD Anderson Cancer Center, \\ Houston, TX, USA \\ ${ }^{6}$ Department of Pathology, The University of Texas MD Anderson Cancer Center, Houston, TX, \\ USA \\ ${ }^{7}$ Morgan Welch Inflammatory Breast Cancer Research Program and Clinic, The University of \\ Texas MD Anderson Cancer Center, Houston, TX, USA \\ ${ }^{8}$ Department of Medical Oncology, The National Cancer Institute, Cairo University, Cairo, Egypt
}

\section{Abstract}

Inflammatory breast cancer (IBC) is a rare and aggressive disease. Previous studies have shown that among patients with stage III breast cancer, IBC is associated with a worse prognosis than noninflammatory breast cancer (non-IBC). Whether this difference holds true among patients with stage IV breast cancer has not been studied. We tested the hypothesis that overall survival (OS) is worse in patients with IBC than in those with non-IBC among patients with distant metastasis at diagnosis (stage IV disease). We reviewed the records of 1504 consecutive patients with stage IV breast cancer (IBC: 206; non-IBC: 1298) treated at our institution from 1987 through 2012. Survival curves for IBC and non-IBC subcohorts were compared. The Cox proportional hazards

Correspondence to: Naoto T. Ueno, nueno@mdanderson. org.

This research was presented in part in December 2013 at the San Antonio Breast Cancer Symposium in San Antonio, Texas.

Conflict of interest The authors declare that they have no conflict of interest. 
model was used to determine predictors of OS. The median follow-up period was 4.7 years. IBC was associated with shorter median OS time than non-IBC (2.27 years vs. 3.40 years; $P=0.0128$, log-rank test). In a multicovariate Cox model that included 1389 patients, the diagnosis of IBC was a significant independent predictor of worse OS (hazard ratio $=1.431, P=0.0011$ ). Other significant predictors of worse OS included Black (vs. White) ethnicity, younger age at diagnosis, negative HER2 status, and visceral (vs. nonvisceral) site of metastasis. IBC is associated with shorter OS than non-IBC in patients with distant metastasis at diagnosis. The prognostic impact of IBC should be taken into consideration among patients with stage IV breast cancer.

\section{Keywords}

Stage IV; Breast cancer; Inflammatory breast cancer; IBC; Prognosis; Metastasis

\section{Introduction}

Inflammatory breast cancer (IBC) is currently defined according to the clinical criteria outlined in the seventh edition of the AJCC Cancer Staging Manual of the American Joint Committee on Cancer (AJCC) [1, 2]. IBC is characterized by diffuse erythema and edema (peaud'orange) of the breast, often without an underlying tumor mass, in the presence of pathologic evidence of breast cancer. Histologic evidence of dermal lymphatic invasion confirms the diagnosis but is not mandatory.

Although IBC accounts for a mere 2- 6\% of all breast cancers in the United States [3- 5], IBC is responsible for a disproportionate $7 \%$ of breast cancer-related deaths [4]. Approximately, 20-30\% of patients with IBC present with distant metastasis at diagnosis (de novo metastasis, classified as stage IV disease) [6-8] compared to 6-10\% of patients with noninflammatory breast cancer (non-IBC) [9, 10]. Distant metastasis remains the main cause of death in patients with breast cancer despite important medical advances [11].

Several retrospective studies have compared IBC to non-IBC among patients with stage III breast cancer [12-14]. These studies have concluded that IBC is associated with worse prognosis than non-IBC in patients without distant metastasis. To date, outcomes of patients with stage IV IBC and stage IV non-IBC have not been compared.

We hypothesized that overall survival (OS) is worse in patients with IBC than in those with non-IBC among patients with distant metastasis at diagnosis. We tested this hypothesis by performing a retrospective comparison of OS between patients presenting with stage IV IBC and stage IV non-IBC.

\section{Patients and methods}

\section{Patient selection}

The Institutional Review Board of The University of Texas MD Anderson Cancer Center approved the protocol for this study (PA13-0117) prior to data collection, and a waiver of informed consent was granted because the study was retrospective. We searched the prospectively maintained Breast Cancer Management System database at MD Anderson 
Cancer Center to identify patients who were diagnosed with breast cancer and metastasis at diagnosis (stage IV) at our institution during the period from August 1987 through March 2012. Patients with stage III disease at initial diagnosis including those who later developed distant metastasis were not included. Stage IV disease was defined according to the 7th edition of the AJCC Cancer Staging Manual [1]. The AJCC defines stage IV disease as clinical evidence of distant metastasis discovered within 4 months after the date of diagnosis or prior to the initial introduction of definitive treatment (surgery, systemic therapy, radiation therapy, active surveillance, or palliative care), whichever comes first, as long as the cancer has not clearly progressed during that time frame [1].

We identified 1898 patients who met these criteria. From this group, we excluded male patients ( $n=15)$, patients with secondary IBC ( $n=3)$, patients with absent follow-up information $(n=1)$, patients diagnosed with stage IV disease on the basis of ipsilateral supraclavicular node involvement alone prior to the 2006 AJCC staging revisions ( $n=226$ ), and patients whose disease did not meet the strict AJCC definition of stage IV breast cancer $(n=149)$ [1]. We defined secondary IBC as IBC that occurred after a history of non-IBC and IBC described as "secondary IBC" in the electronic medical record. In total, 1504 patients (IBC: 206; non-IBC: 1298) were included in the analysis. Patients with de novo metastatic IBC represented approximately $20 \%$ of all patients with IBC in the Breast Cancer Management System database.

All patients in this study were diagnosed by a multidisciplinary team composed of a breast oncologist, a surgeon, and a radiation oncologist. All pathologic specimens were reviewed by a breast pathologist at our institution. As part of the routine staging workup, all patients had a complete history and physical examination, blood cell counts, serum chemistry tests, bilateral mammography, ultrasonography, chest radiography, and bone scan. A liver-spleen scan was performed routinely before computed tomography of the abdomen and pelvis became available. Positron emission tomography/computed tomography was considered optional according to guideline recommendations and was performed for some patients [15]. For this study, a clinical diagnosis of IBC was defined according to the criteria outlined in the seventh edition of the AJCC Cancer Staging Manual and the recommendations of the international expert panel on IBC [1, 2]. Patients with onset of erythema more than 6 months after breast cancer diagnosis were considered to have neglected, locally advanced breast cancer with secondary erythema and were not considered to have IBC [2]. Patients with a diagnosis of IBC that was not clinically confirmed (e.g., "probably IBC") were not considered to have IBC in the final analysis (42 of 248 patients originally classified as having IBC).

The burden of distant metastatic disease was assessed by calculating the number of sites of metastasis. Multiple sites of metastasis were defined as metastasis involving two or more organ sites. Visceral metastasis was defined as metastasis to the liver, lungs, brain, or other organs; nonvisceral metastasis was defined as bone-only disease and/or metastasis to the distant soft tissue and/or lymphatics.

Estrogen receptor status, progesterone receptor status, and HER2 status were determined using immunohistochemical (IHC) analysis according to the American Society of Clinical 
Oncology-College of American Pathologists recommendations [16, 17]. Hormone receptor status was considered positive if a tumor was positive for estrogen receptor and/or progesterone receptor and negative if a tumor was negative for both receptors. HER2 status was considered positive if receptor expression was classified as +3 on IHC staining or the fluorescent in situ hybridization result was positive. HER2 status was considered negative if receptor expression was classified as 0 or +1 on IHC staining or was classified as +2 and fluorescent in situ hybridization results were nonamplified.

Treatment decisions were made by the treating physician, and treatment commonly incorporated anthracycline-based or taxane-based chemotherapy regimens and HER2targeting therapy when indicated. A significant proportion of patients with stage IV breast cancer underwent locoregional surgery, defined as surgical excision of the primary tumor (mastectomy or breast-conserving surgery) and/or regional lymph nodes. Patients who underwent only breast biopsy for tissue diagnosis were categorized as not having had surgery.

\section{Statistical methods}

Patients were categorized according to IBC status. The primary endpoint was OS time calculated from the date of diagnosis to the date of death due to any cause or the date of last follow-up (censored). The variables of interest are listed in Table 1. Patient characteristics were first summarized using standard descriptive statistics and frequency tabulation. Age at diagnosis between IBC and non-IBC patients was compared using Wilcoxon rank sum test. Associations between IBC status and patient/tumor characteristics were assessed via crosstabulation and $\chi^{2}$-test or Fisher's exact test. The median follow-up time was calculated using the reverse Kaplan-Meier estimator [18]. OS was estimated using the Kaplan-Meier method, and comparisons between or among patient characteristics were assessed using logrank test. Multicovariate Cox proportional hazards models were used to assess the effect of covariates of interest on OS. The proportional hazards assumption was checked for all covariates in the final models. The stratified Cox model was used to handle the covariates that violated the proportional hazards assumption. The double robust estimation method, which combines propensity-score matching with direct regression on the outcomes, was used to conduct a propensity-score matched analysis [19, 20]. All computations were carried out in SAS 9.3 (SAS Institute Inc., Cary, NC, USA) and Splus 8.2 (TIBCO Software Inc, Palo Alto, California).

\section{Results}

\section{Study population}

The study included 1504 patients who presented with stage IV breast cancer, 206 with IBC and 1298 with non-IBC. The median follow-up period was 4.7 years (95\% CI 4.1-5.5). Baseline characteristics of these two patient groups are summarized in Table 1. Patients with IBC were more likely to be premenopausal $(P=0.0022)$, present with better performance status $(P=0.0031)$, and have tumors with HER2 amplification $(P=0.02)$ and triplereceptor-negative status $(P<0.0001)$. A considerably lower proportion of patients with IBC than non-IBC presented with hormone-receptor-positive tumors (52.2 vs. $76.4 \%, P<$ 
0.0001). Patients with IBC presented more frequently than patients with non-IBC with nonvisceral metastasis at diagnosis, particularly in the form of distant nodal/soft tissue metastasis $(P=0.0341)$. Moreover, patients with IBC presented more frequently with multiple sites of distant metastasis ( 49.5 vs. $41.3 \%, P=0.0266)$.

The vast majority $(90.8 \%)$ of patients with IBC were treated with chemotherapy as the initial line of treatment, compared to $57.9 \%$ of patients with non-IBC $(P<0.0001)$. Among patients with HER2-positive tumors, patients with IBC were more likely to receive HER2targeting therapy (mainly trastuzumab and/or lapatinib) as part of their initial treatment plan than were patients with non-IBC ( 65.7 vs. $52.6 \%, P=0.0457)$. More patients with IBC than non-IBC were treated with locoregional surgery (39.3 vs. $24.3 \%, P<0.0001)$.

\section{Overall survival}

The total number of recorded events (deaths) was 882 . The median OS time was shorter for patients with IBC than for those with non-IBC: 2.27 years (95\% CI 1.92-2.88) versus 3.40 years (95\% CI 3.20-3.68) $(P=0.0128$, log-rank test) (Fig. 1$)$. The 2- and 5-year OS rates for various patient categories for IBC and non-IBC separately are presented in Table 2.

In univariate analysis, the hazard ratios (HRs) for OS between patients with IBC and patients with non-IBC differed between groups defined on the basis of age, ethnicity, HER2 status, and receipt of surgery or HER2-targeting therapy (Fig. 2). These differences in HR were particularly pronounced in categories that included characteristics known to be associated with better prognosis, such as better ECOG performance status ( 0 or 1 vs. > 1), a single metastatic site (vs. multiple metastases), and nonvisceral (vs. visceral) metastasis.

Among patients with triple-negative breast cancer, there was no significant difference in OS between patients with IBC and those with non-IBC (HR for IBC $=1.31$; $95 \%$ CI 0.94 1.82). Likewise, among patients who received up-front HER2-targeting therapy, there was no significant difference in OS between patients with IBC and those with non-IBC (HR for IBC vs. non-IBC $=1.06 ; 95 \%$ CI 0.61-1.82). Similarly, among patients with hormonereceptor-positive tumors, there was no significant difference in OS between the IBC and non-IBC groups (Fig. 2). However, in the subgroup of patients with hormone-receptor positive, HER2-negative tumors, there was a trend for shorter OS associated with IBC compared to non-IBC (HR $=1.38 ; 95 \%$ CI 1.00-1.91).

Results from two multicovariate Cox models that included 1389 patients are presented in Table 3 (without stratification) and Table 4 (stratified by variables that did not satisfy the proportional hazards assumption). The Cox models included 1389 patients because 115 patients ( $7.6 \%$ of the 1504 patients in the study) were excluded because of missing data. The vast majority of cases of missing data were due to lack of HER 2 testing prior to the approval of trastuzumab in 1998. After stratification by ECOG performance status, hormone receptor status, number of metastatic sites (multiple vs. single), locoregional surgery, and hormonal therapy, IBC was significantly associated with shorter OS time than non-IBC (HR $=1.431, P=0.0011)$ after adjustment for other significant factors. Results of the propensityscore matched analysis were consistent with this conclusion: HR $($ IBC vs. non-IBC) $=1.342$ $(P=0.0275)$. 


\section{Discussion}

Our findings show that among patients with breast cancer and distant metastasis at diagnosis (stage IV disease), patients with IBC have worse OS than patients with non-IBC. This poor outcome among patients with IBC relative to patients with non-IBC was reflected in shorter median OS time, lower 2-year and 5-year OS rates, results of a propensity-score matched analysis, and the HR on multivariate analysis $(1.431 ; P=0.0011)$.

The median survival of breast cancer patients with distant metastasis at diagnosis has been reported to range from 18 to 40 months depending on the predominant pattern of metastasis, number of metastatic sites, ECOG performance status, and tumor receptor status [21, 22]. Noncomparative studies have also explored the outcome of patients with de novo metastatic IBC. Dawood et al. showed using data from the Surveillance, Epidemiology and End Results database that among patients with IBC, women with de novo metastasis had significantly lower 2-year IBC-specific survival and OS rates than women with stage IIIB or IIIC disease [23]. The authors identified several predictors of more favorable prognosis, including White ethnicity, tumors expressing hormone receptors, lower tumor grade, and receipt of locoregional surgery and/or radiation therapy. Two other studies reported similar outcomes among patients with de novo metastatic IBC [24, 25].

Breast cancer subtypes differ considerably between IBC and non-IBC, and these differences could directly impact OS or could impact treatment options, which could in turn impact OS. In this study, we found that patients with IBC were more likely to present with triplenegative breast cancer; however, among patients presenting with triple-negative breast cancer, there was no difference in OS between patients with IBC and those with non-IBC. This seems consistent with the findings of another study that examined triple-negative tumors and was unable to show differences between IBC and non-IBC in terms of outcome or at the mRNA expression level [26].

Likewise, patients with IBC were more likely to present with HER2-positive tumors. Historically, HER2 overexpression was associated with poor prognosis in patients with breast tumors [27, 28]. However, HER2-targeting therapy has altered the natural history of HER2-positive breast cancer so that this disease is now associated with a better prognosis [29]. In this study, HER 2 positivity conferred a better overall prognosis in multivariate analysis (Table $4 ; \mathrm{HR}=0.659 ; 95 \% \mathrm{CI} 0.553-0.786$ ). Moreover, among patients who received up-front HER2-targeting therapy, OS was not different between IBC and non-IBC (HR for IBC vs. non-IBC $=1.06 ; 95 \%$ CI 0.61-1.82).

We found that patients with IBC were less likely to present with hormone-receptor-positive disease. Previous evidence in the non-metastatic setting suggests that among patients with hormone-receptor-positive disease, patients with IBC have worse outcomes than patients with non-IBC [30, 31]. Our results show that there was no survival difference between the two cohorts among patients with hormone-receptor-positive tumors (Fig. 2). Although in our study, some differences in outcome were observed among smaller subgroups (hormonereceptor-positive, HER2-negative tumors, and patients who received hormonal treatment as initial therapy), it is important to be cautious in interpreting univariate differences between 
these small subgroups, given the absence of a clear understanding of the molecular differences between IBC and non-IBC.

Up-front chemotherapy did not appear to offer any OS benefit in our study, although locoregional surgery was associated with better OS rates in both IBC and non-IBC (Table 2). We found that the OS of patients treated with up-front chemotherapy did not differ from the survival of those who were treated with other therapeutic modalities as first-line therapy. Additionally, the type of chemotherapy regimen used (anthracycline based or taxane based) did not affect OS. The long-term effects of first-line chemotherapy in metastatic breast cancer are known to be subject to confounding by the effects of subsequent therapies [32]. Patients in the IBC group were more likely to undergo locoregional surgery than were patients in the non-IBC group (39.3 vs. $24.3 \%, P<0.0001)$. Recent reports by other groups indicate that an increasingly high percentage of patients with metastatic breast cancer are being offered surgery (ranging from 37 to $61.3 \%$ ), mainly because of a lack of therapeutic options [32,33]. In the present study, 2-year and 5-year OS rates were significantly better in patients (IBC and non-IBC) who underwent surgery than in those who did not. Similar observations were reported from the Surveillance, Epidemiology and End Results database [23]. Benefit from surgery in patients presenting with metastatic disease remains controversial and may reflect a selection bias for patients who present with less debilitating disease, better performance status, or higher chemosensitivity [33, 34].

Although OS of patients with newly diagnosed stage IV breast cancer has improved over time with the introduction of new therapies (e.g., taxanes, aromatase inhibitors, and HER2targeting therapy), the impact of such therapies on the outcome of patients with IBC has been less clear [21, 35, 36]. As there is no specific therapy for stage IV IBC, therapy for IBC is extrapolated from therapy for non-IBC, and novel therapeutic agents are introduced during the same time period for these subtypes of disease. In our study, there was no OS difference between patients with IBC and those with non-IBC who received novel therapies (taxanes, HER2-targeting therapy) or had hormone-receptor positive disease (Fig. 2). We also examined the impact of the launch of a dedicated IBC clinic (October 2006) on the OS of patients with stage IV IBC and observed no improvement in OS after the clinic launch (data not shown) [36].

Our study has several strengths. We believe this is the first study to compare long-term OS of patients with IBC and non-IBC with distant metastasis at diagnosis. To our knowledge, it is also the largest cohort to date of patients with newly diagnosed stage IV breast cancer treated at a single institution. Our analysis offers unique information about the outcome and prognosis of patients with IBC presenting with distant metastasis at diagnosis. We assessed the impact of several covariates that had not been assessed in previous studies concerning de novo metastatic IBC. We believe that our results suggest that IBC is not a surrogate for aggressive subtypes of breast cancer but is an independent prognostic factor. Limitations of our study include its retrospective nature and the inherent biases in patient and treatment selection at a single institution. However, given that de novo metastatic IBC is very rare, the question of whether IBC is associated with worse OS than non-IBC among patients with distant metastasis at diagnosis is unlikely to be resolved via prospective studies. 
In conclusion, our findings indicate that patients with IBC and distant metastasis at diagnosis have worse survival than stage-matched patients with non-IBC. By demonstrating the prognostic impact of IBC in stage IV disease, this study could potentially have a large clinical impact on the staging and management of breast cancer.

\section{Acknowledgments}

This work was supported in part by a State of Texas Rare and Aggressive Breast Cancer Research Program grant; the National Institutes of Health/National Cancer Institute under award number P30CA016672, which supports the Biostatistics Shared Resource; and grant R01CA07466 from the National Cancer Institute (to Y.S.). We thank Jie Willey, MSN, and Mr. Limin Hsu of the Department of Breast Medical Oncology, The University of Texas MD Anderson Cancer Center, for their role in the preparation of this protocol and data management and Stephanie Deming of the Department of Scientific Publications, MD Anderson, for editing this manuscript.

\section{References}

1. Edge, S.; Byrd, D.; Compton, C. American Joint Committee on Cancer (AJCC) staging handbook. New York: Springer; 2010.

2. Dawood S, Merajver SD, Viens P, et al. International expert panel on inflammatory breast cancer: consensus statement for standardized diagnosis and treatment. Ann Oncol. 2011; 22:515-523. [PubMed: 20603440]

3. Anderson WF, Schairer C, Chen BE, et al. Epidemiology of inflammatory breast cancer (IBC). Breast Dis. 2005; 22:9-23. [PubMed: 16735783]

4. Hance KW, Anderson WF, Devesa SS, et al. Trends in inflammatory breast carcinoma incidence and survival: the surveillance, epidemiology, and end results program at the National Cancer Institute. J Natl Cancer Inst. 2005; 97:966-975. [PubMed: 15998949]

5. Jaiyesimi IA, Buzdar AU, Hortobagyi G. Inflammatory breast cancer: a review. J Clin Oncol. 1992; 10:1014-1024. [PubMed: 1588366]

6. Wingo PA, Jamison PM, Young JL, Gargiullo P. Population-based statistics for women diagnosed with inflammatory breast cancer (United States). Cancer Causes Control. 2004; 15:321-328. [PubMed: 15090727]

7. Elias EG, Vachon DA, Didolkar MS, Aisner J. Long-term results of a combined modality approach in treating inflammatory carcinoma of the breast. Am J Surg. 1991; 162:231-235. [PubMed: 1656805]

8. Curcio LD, Rupp E, Williams WL, et al. Beyond palliative mastectomy in inflammatory breast cancer-a reassessment of margin status. Ann Surg Oncol. 1999; 6:249-254. [PubMed: 10340883]

9. Miller KD, Sledge GW Jr. The role of chemotherapy for metastatic breast cancer. Hematol Oncol Clin N Am. 1999; 13:415-434.

10. O'Shaughnessy J. Extending survival with chemotherapy in metastatic breast cancer. Oncologist. 2005; 10(Suppl 3):20-29. [PubMed: 16368868]

11. Lu J, Steeg PS, Price JE, et al. Breast cancer metastasis: challenges and opportunities. Cancer Res. 2009; 69:4951-4953. [PubMed: 19470768]

12. Cristofanilli M, Valero V, Buzdar AU, et al. Inflammatory breast cancer (IBC) and patterns of recurrence: understanding the biology of a unique disease. Cancer. 2007; 110:1436-1444. [PubMed: 17694554]

13. Dawood S, Ueno NT, Valero V, et al. Differences in survival among women with stage III inflammatory and noninflammatory locally advanced breast cancer appear early: a large population-based study. Cancer. 2011; 117:1819-1826. [PubMed: 21509759]

14. Low JA, Berman AW, Steinberg SM, et al. Long-term follow-up for locally advanced and inflammatory breast cancer patients treated with multimodality therapy. J Clin Oncol. 2004; 22:4067-4074. [PubMed: 15483018]

15. National Comprehensive Cancer Network. NCCN guidelines, version 3.2014, invasive breast cancer. BINV-17. 2014. 
16. Hammond ME, Hayes DF, Dowsett M, et al. American Society of Clinical Oncology/College Of American Pathologists guideline recommendations for immunohistochemical testing of estrogen and progesterone receptors in breast cancer. J Clin Oncol. 2010; 28:2784-2795. [PubMed: 20404251]

17. Wolff AC, Hammond ME, Hicks DG, et al. Recommendations for human epidermal growth factor receptor 2 testing in breast cancer: American Society of Clinical Oncology/College of American Pathologists clinical practice guideline update. J Clin Oncol. 2013; 31:3997-4013. [PubMed: 24101045]

18. Schemper M, Smith TL. A note on quantifying follow-up in studies of failure time. Control Clin Trials. 1996; 17:343-346. [PubMed: 8889347]

19. Robins JM, Rotnitzky A. Comment on the Bickel and Kwon Article, "Inference for semiparametric models: some questions and an answer.". Stat Sin. 2001; 11:920-936.

20. Austin PC. The performance of different propensity-score methods for estimating relative risks. J Clin Epidemiol. 2008; 61:537-545. [PubMed: 18471657]

21. Andre F, Slimane K, Bachelot T, et al. Breast cancer with synchronous metastases: trends in survival during a 14-year period. J Clin Oncol. 2004; 22:3302-3308. [PubMed: 15310773]

22. Campone M, Bachelot T, Gnant M, et al. Effect of visceral metastases on the efficacy and safety of everolimus in postmenopausal women with advanced breast cancer: subgroup analysis from the BOLERO-2 study. Eur J Cancer. 2013; 49:2621-2632. [PubMed: 23735704]

23. Dawood S, Ueno NT, Valero V, et al. Identifying factors that impact survival among women with inflammatory breast cancer. Ann Oncol. 2012; 23:870-875. [PubMed: 21765048]

24. Sutherland S, Ashley S, Walsh G, et al. Inflammatory breast cancer-the Royal Marsden Hospital experience: a review of 155 patients treated from 1990 to 2007. Cancer. 2010; 116:2815-2820. [PubMed: 20503413]

25. Panades M, Olivotto IA, Speers $\mathrm{CH}$, et al. Evolving treatment strategies for inflammatory breast cancer: a population-based survival analysis. J Clin Oncol. 2005; 23:1941-1950. [PubMed: 15774787]

26. Masuda H, Baggerly KA, Wang Y, et al. Comparison of molecular subtype distribution in triplenegative inflammatory and non-inflammatory breast cancers. Breast Cancer Res. 2013; 15:R112. [PubMed: 24274653]

27. Slamon DJ, Clark GM, Wong SG, et al. Human breast cancer: correlation of relapse and survival with amplification of the HER-2/neu oncogene. Science. 1987; 235:177-182. [PubMed: 3798106]

28. Seshadri R, Firgaira FA, Horsfall DJ, et al. Clinical significance of HER-2/neu oncogene amplification in primary breast cancer. The South Australian Breast Cancer Study Group. J Clin Oncol. 1993; 11:1936-1942. [PubMed: 8105035]

29. Dawood S, Broglio K, Buzdar AU, et al. Prognosis of women with metastatic breast cancer by HER2 status and trastuzumab treatment: an institutional-based review. J Clin Oncol. 2010; 28:9298. [PubMed: 19933921]

30. Iwamoto T, Bianchini G, Qi Y, et al. Different gene expressions are associated with the different molecular subtypes of inflammatory breast cancer. Breast Cancer Res Treat. 2011; 125:785-795. [PubMed: 21153052]

31. Iwamoto T, Bianchini G, Booser D, et al. Gene pathways associated with prognosis and chemotherapy sensitivity in molecular subtypes of breast cancer. J Natl Cancer Inst. 2011; 103:264-272. [PubMed: 21191116]

32. Beslija S, Bonneterre J, Burstein HJ, et al. Third consensus on medical treatment of metastatic breast cancer. Ann Oncol. 2009; 20:1771-1785. [PubMed: 19608616]

33. Khan SA. Does resection of an intact breast primary improve survival in metastatic breast cancer? Oncology (Williston Park). 2007; 21:924-931. discussion 931-922, 934, 942, passim. [PubMed: 17715695]

34. Morrow M, Goldstein L. Surgery of the primary tumor in metastatic breast cancer: closing the barn door after the horse has bolted? J Clin Oncol. 2006; 24:2694-2696. [PubMed: 16702578]

35. Gonzalez-Angulo AM, Hennessy BT, Broglio K, et al. Trends for inflammatory breast cancer: is survival improving? Oncologist. 2007; 12:904-912. [PubMed: 17766649] 
36. Tsai CJ, Li J, Gonzalez-Angulo AM, et al. Outcomes after multidisciplinary treatment of inflammatory breast cancer in the era of neoadjuvant HER2-directed therapy. Am J Clin Oncol. 2013; 38:242-247. [PubMed: 23648437] 


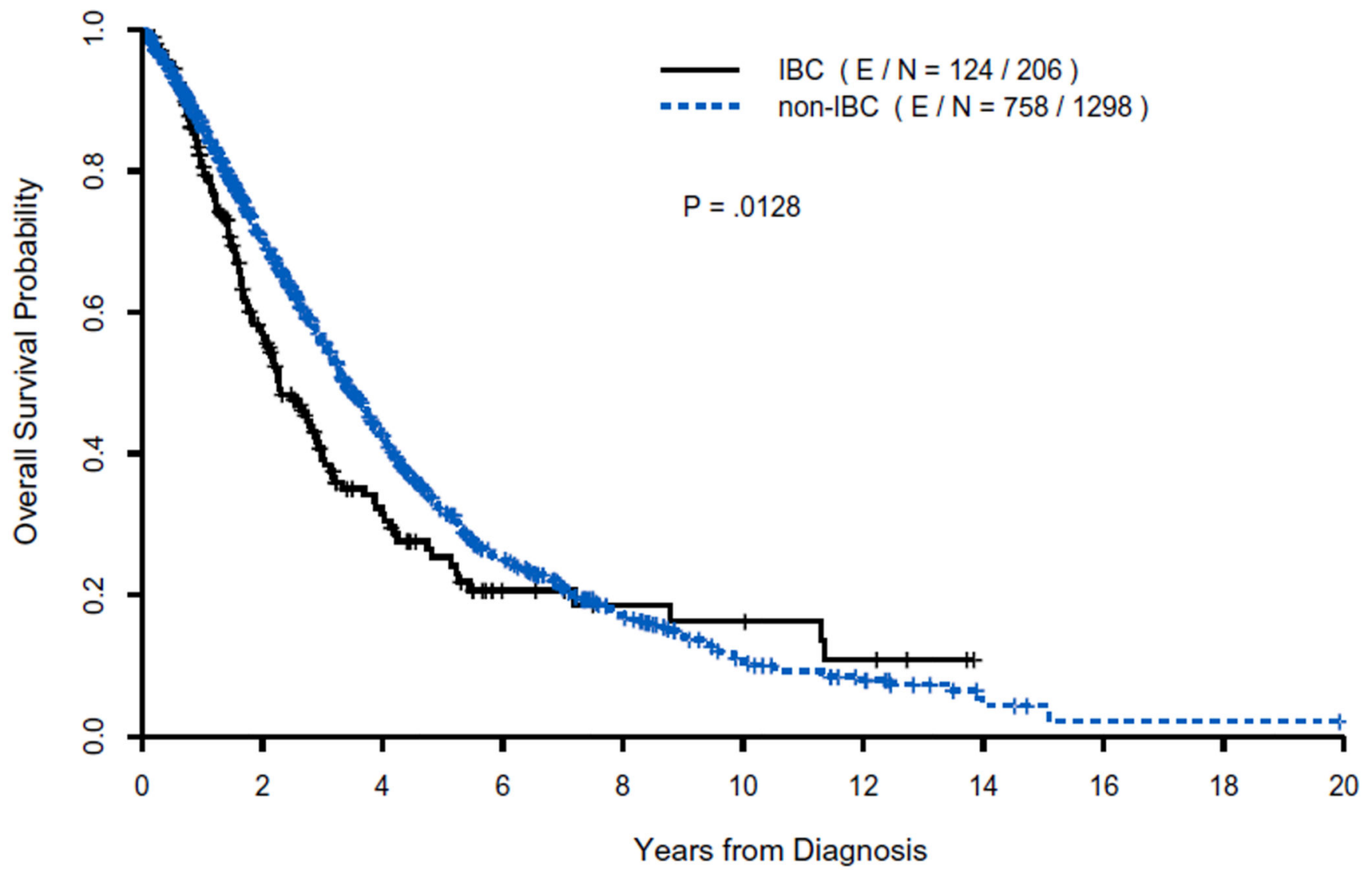

No. at Risk

$\begin{array}{lcccccccc}\text { Non-IBC } & 1298 & 682 & 314 & 144 & 73 & 30 & 17 & 4 \\ \text { IBC } & 206 & 89 & 34 & 12 & 8 & 7 & 4 & 0\end{array}$

Fig. 1.

Overall survival of patients with IBC and non-IBC presenting with distant metastasis at diagnosis. $E / N$, cumulative number of events/total number of patients at risk at time zero 


\begin{tabular}{|c|c|}
\hline Patient Groups & No. Patients \\
\hline All Patients & 1504 \\
\hline $\begin{array}{l}\text { Ethnicity } \\
\text { White } \\
\text { Black } \\
\text { Other }\end{array}$ & $\begin{array}{r}1041 \\
236 \\
227\end{array}$ \\
\hline $\begin{array}{r}\text { Age, years } \\
<65 \\
>=65\end{array}$ & $\begin{array}{r}1217 \\
287\end{array}$ \\
\hline $\begin{array}{c}\text { ECOG performanc } \\
0 \text { or } 1 \\
>1\end{array}$ & $\begin{array}{r}1397 \\
94\end{array}$ \\
\hline $\begin{array}{c}\text { Hormone receptor } \\
\text { Positive } \\
\text { Negative }\end{array}$ & $\begin{array}{r}1065 \\
393\end{array}$ \\
\hline $\begin{array}{l}\text { HER2 status } \\
\text { Positive } \\
\text { Negative }\end{array}$ & $\begin{array}{r}395 \\
1001\end{array}$ \\
\hline $\begin{array}{c}\text { No. of metastatic s } \\
1 \\
>1\end{array}$ & $\begin{array}{l}866 \\
638\end{array}$ \\
\hline $\begin{array}{c}\text { Site of metastasis } \\
\text { Visceral } \\
\text { Nonvisceral }\end{array}$ & $\begin{array}{l}847 \\
657\end{array}$ \\
\hline $\begin{array}{c}\text { Locoregional surge } \\
\text { Yes } \\
\text { No }\end{array}$ & $\begin{array}{r}397 \\
1107\end{array}$ \\
\hline $\begin{array}{l}\text { HER2-targeting the } \\
\text { Yes } \\
\text { No }\end{array}$ & $\begin{array}{r}219 \\
1285\end{array}$ \\
\hline $\begin{array}{c}\text { Hormonal therapy } \\
\text { Yes } \\
\text { No }\end{array}$ & $\begin{array}{r}453 \\
1051\end{array}$ \\
\hline
\end{tabular}

Hazard Ratio (IBC vs Non-IBC)

$1.27(1.05-1.54)$

p-value

04

041

236

1217

287

397

065

295

1001

866

638

847
657

397

1107

219

285

453
1051

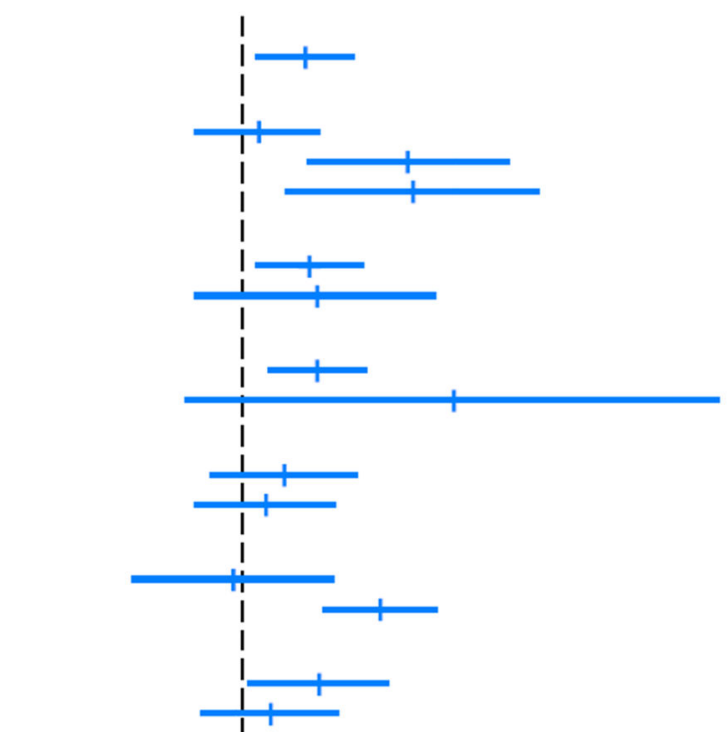

$1.06(0.83-1.35)$

$1.89(1.28-2.81)$

$1.93(1.18-3.15)$

$1.29(1.05-1.60)$

$1.33(0.83-2.12)$

$1.33(1.10-1.62)$

$2.26(0.80-6.36)$

$1.17(0.88-1.56)$

$1.09(0.83-1.43)$

$0.96(0.65-1.42)$

$1.70(1.36-2.13)$

$1.34(1.02-1.76)$

$1.11(0.85-1.45)$

$1.23(0.94-1.61)$

$1.45(1.10-1.90)$

$1.10(0.76-1.60)$

$1.83(1.46-2.29)$

$1.06(0.61-1.82)$

$1.36(1.11-1.67)$

$3.48(1.53-7.92)$

$1.12(0.92-1.37)$
0.013

0.656

0.001

0.009

0.015

0.238

0.004

0.124

0.280

0.548

0.855

$<0.001$

0.035

0.463

0.124

0.008

0.604 $<0.001$

0.840 0.003

0.003 0.256

Fig. 2.

Forest plot of hazard ratio for death for IBC versus non-IBC among patient groups defined on the basis of different clinicopathologic and treatment characteristics. Statistically significant interactions were observed between IBC status and HER2 status, and between IBC status and upfront hormonal therapy $(P=0.0262$ and $P=0.0222$, respectively), when including the interaction terms in the stratified multicovariate model 
Table 1

Patient characteristics by IBC status

\begin{tabular}{|c|c|c|c|}
\hline Characteristic & $\begin{array}{l}\text { IBC } \\
(n=206)\end{array}$ & $\begin{array}{l}\text { Non-IBC } \\
(n=1298)\end{array}$ & $P$ value \\
\hline Age at diagnosis (years) & & & 0.0016 \\
\hline Mean \pm SD & $50.35 \pm 11.73$ & $53.39 \pm 12.83$ & \\
\hline Median (range) & $49.5(26-85)$ & $53(17-93)$ & \\
\hline Ethnicity & & & 0.5299 \\
\hline White & $145(70.4)$ & $896(69)$ & \\
\hline Black & $35(17)$ & $201(15.5)$ & \\
\hline Other & $26(12.6)$ & $201(15.5)$ & \\
\hline Menopausal status & & & 0.0022 \\
\hline Postmenopausal & $102(50.2)$ & $793(61.6)$ & \\
\hline Premenopausal & $101(49.8)$ & $495(38.4)$ & \\
\hline Missing & 3 & 10 & \\
\hline ECOG performance status & & & 0.0031 \\
\hline 0 or 1 & $201(98)$ & $1196(93)$ & \\
\hline$>1$ & $4(2)$ & $90(7)$ & \\
\hline Missing & 1 & 12 & \\
\hline Hormone receptor status & & & $<0.0001$ \\
\hline Positive & $105(52.2)$ & $960(76.4)$ & \\
\hline Negative & $96(47.8)$ & $297(23.6)$ & \\
\hline Missing & 5 & 41 & \\
\hline HER2 status & & & 0.0200 \\
\hline Positive & $70(35.2)$ & $325(27.2)$ & \\
\hline Negative & $129(64.8)$ & $872(72.8)$ & \\
\hline Missing & 7 & 101 & \\
\hline Subtype & & & $<0.0001$ \\
\hline HR +/HER2+ & $34(17.3)$ & $215(18)$ & \\
\hline HR +/HER2- & $70(35.5)$ & $692(58.1)$ & \\
\hline HR -/HER2+ & $35(17.8)$ & $107(9)$ & \\
\hline HR -/HER2- & $58(29.4)$ & $178(14.9)$ & \\
\hline Missing & 9 & 106 & \\
\hline No. of metastatic sites & & & 0.0266 \\
\hline 1 & $104(50.5)$ & $762(58.7)$ & \\
\hline$>1$ & $102(49.5)$ & $536(41.3)$ & \\
\hline Site of metastasis & & & 0.0341 \\
\hline Visceral & $102(49.5)$ & $745(57.4)$ & \\
\hline Nonvisceral & $104(50.5)$ & $553(42.6)$ & \\
\hline Chemotherapy as first-line therapy ${ }^{a}$ & & & $<0.0001$ \\
\hline Yes & $187(90.8)$ & $751(57.9)$ & \\
\hline No & $19(9.2)$ & $547(42.1)$ & \\
\hline
\end{tabular}




\begin{tabular}{|c|c|c|c|c|}
\hline \multirow{8}{*}{ 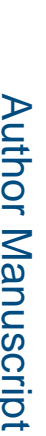 } & Characteristic & $\begin{array}{l}\text { IBC } \\
(n=206)\end{array}$ & $\begin{array}{l}\text { Non-IBC } \\
(n=1298)\end{array}$ & $P$ value \\
\hline & Anthracycline therapy ${ }^{a}$ & & & $<0.0001$ \\
\hline & Yes & 94 (45.6) & $353(27.2)$ & \\
\hline & No & $112(54.4)$ & $945(72.8)$ & \\
\hline & Taxane therapy ${ }^{a}$ & & & $<0.0001$ \\
\hline & Yes & $132(64.1)$ & $465(35.8)$ & \\
\hline & No & $74(35.9)$ & $833(64.2)$ & \\
\hline & HER2-targeting therapy $a$ & & & 0.0007 \\
\hline & Yes & $46(22.3)$ & $173(13.3)$ & \\
\hline & No & $160(77.7)$ & $1125(86.7)$ & \\
\hline & Hormonal therapy ${ }^{a}$ & & & $<0.0001$ \\
\hline & Yes & $9(4.4)$ & $444(34.2)$ & \\
\hline & No & 197 (95.6) & $854(65.8)$ & \\
\hline ह & Locoregional surgery & & & $<0.0001$ \\
\hline 음 & Yes & $81(39.3)$ & $316(24.3)$ & \\
\hline$\frac{\pi}{z}$ & No & $125(60.7)$ & $982(75.7)$ & \\
\hline
\end{tabular}

Values in table are number of patients (percentage) unless otherwise indicated

$H R$ hormone receptor

$a_{\text {Therapy received as first-line }}$ 


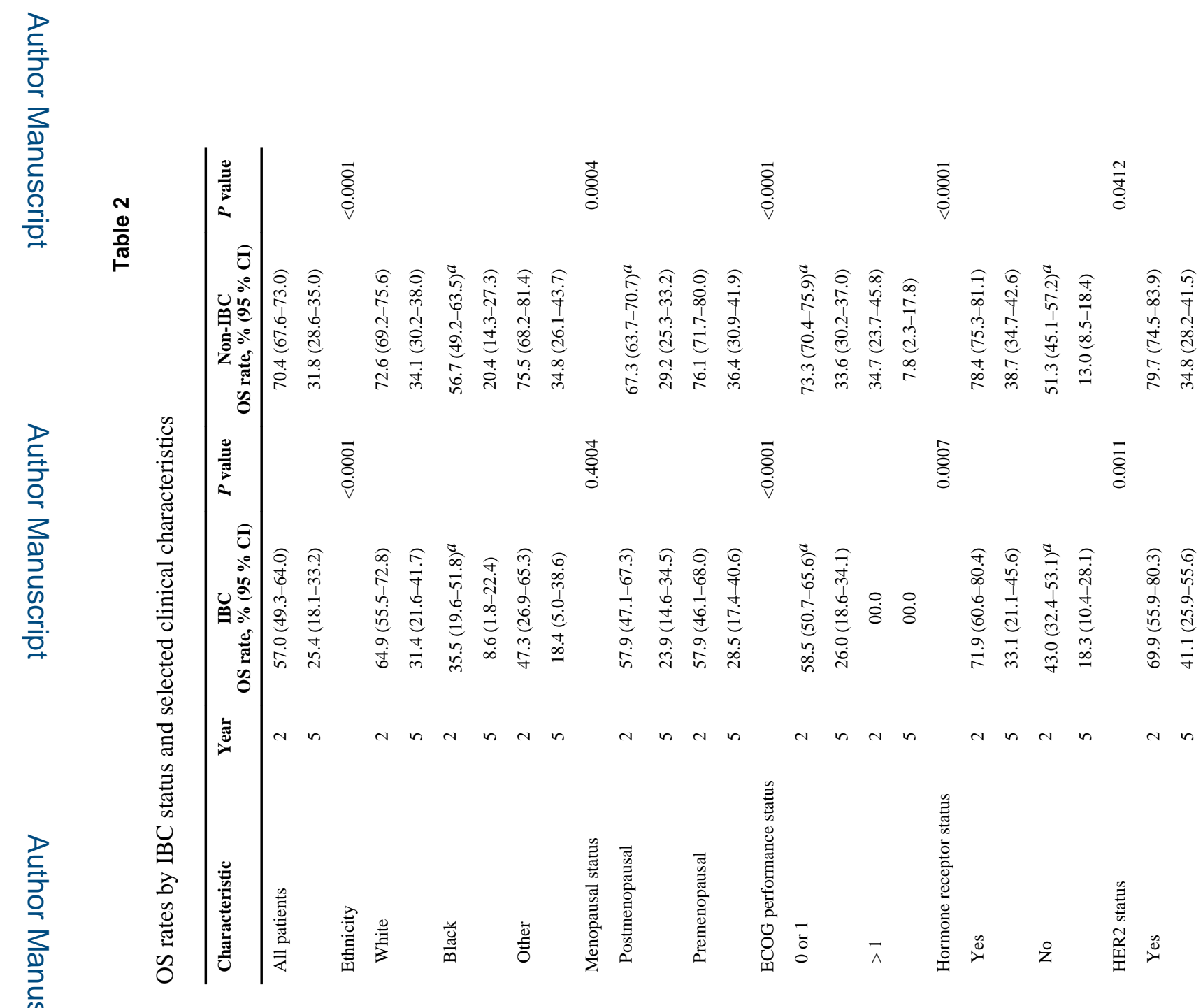
Breast Cancer Res Treat. Author manuscript; available in PMC 2016 July 01. 
Fouad et al.

Page 16
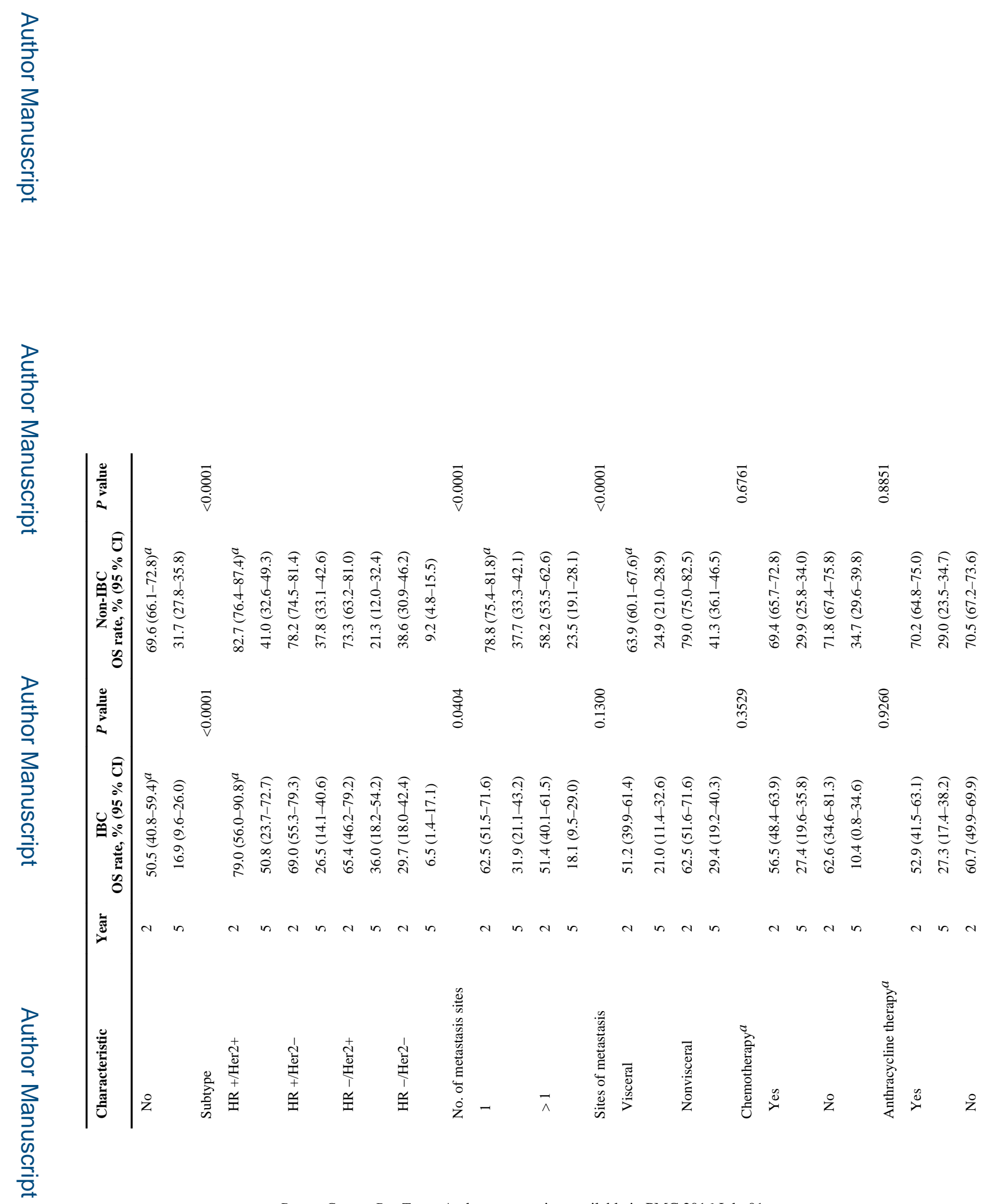

Breast Cancer Res Treat. Author manuscript; available in PMC 2016 July 01. 


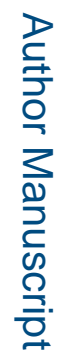

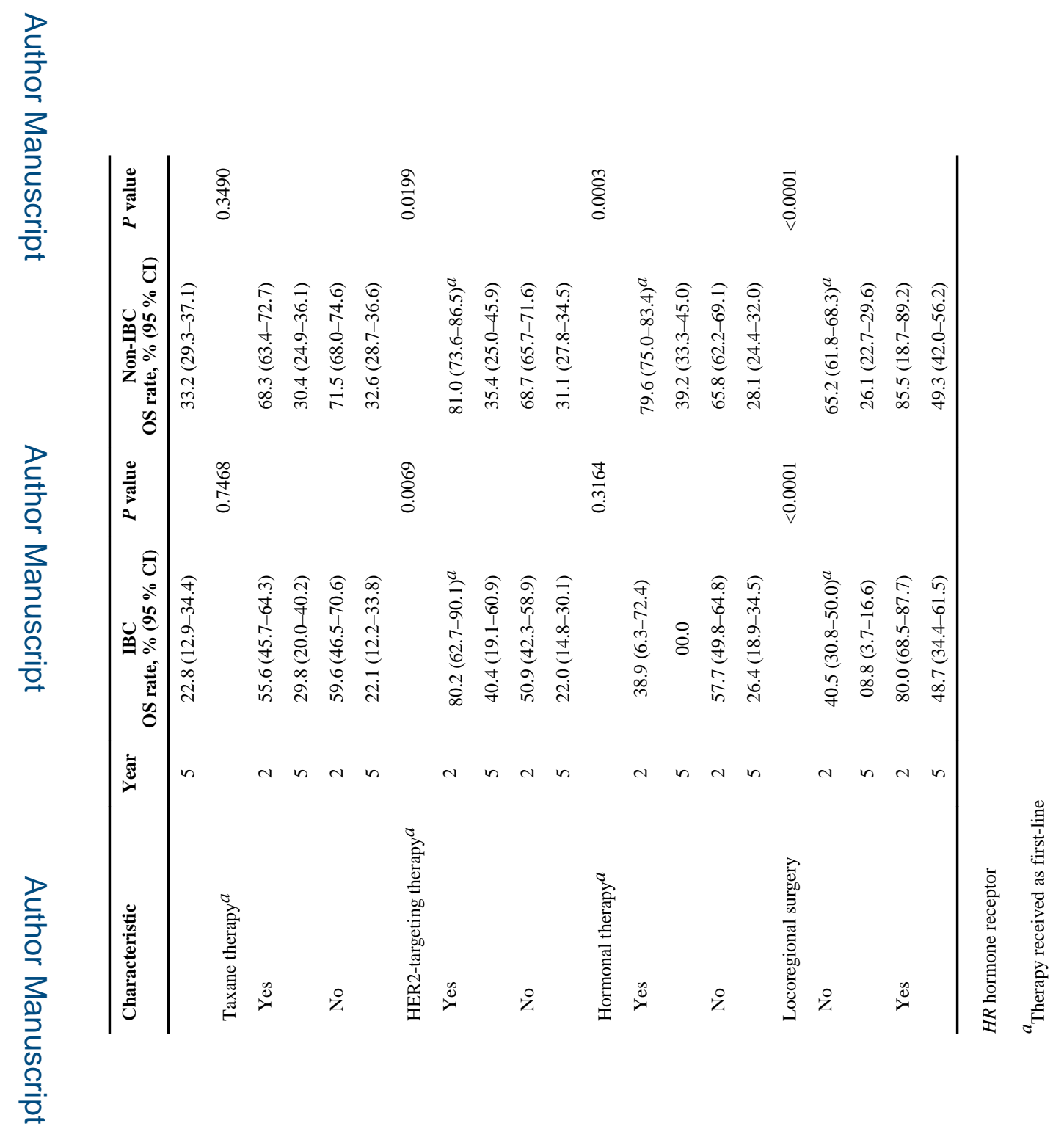

Breast Cancer Res Treat. Author manuscript; available in PMC 2016 July 01. 
Table 3

Multicovariate Cox regression model for overall survival without stratification

\begin{tabular}{|c|c|c|c|}
\hline Parameter & Hazard ratio & $95 \% \mathrm{CI}$ & $P$ value \\
\hline IBC versus non-IBC & 1.406 & $1.137-1.740$ & 0.0017 \\
\hline \multicolumn{4}{|l|}{ Ethnicity } \\
\hline Black versus White & 1.489 & $1.243-1.784$ & $<0.0001$ \\
\hline Other versus White & 1.207 & $0.977-1.491$ & 0.0819 \\
\hline Age at diagnosis & 1.007 & $1.001-1.013$ & 0.0336 \\
\hline ECOG performance status $>1$ versus 0 or $1^{a}$ & 3.017 & $2.300-3.958$ & $<0.0001$ \\
\hline Hormone receptor positive versus negative ${ }^{a}$ & 0.500 & $0.422-0.594$ & $<0.0001$ \\
\hline HER2 positive versus negative & 0.635 & $0.536-0.752$ & $<0.0001$ \\
\hline Multiple metastases versus single metastasis ${ }^{a}$ & 1.278 & $1.082-1.509$ & 0.0059 \\
\hline Visceral versus nonvisceral metastases & 1.226 & $1.035-1.453$ & 0.0184 \\
\hline Locoregional surgery versus no locoregional surgery $a$ & 0.399 & $0.331-0.481$ & $<0.0001$ \\
\hline Hormonal therapy versus no hormonal therapy ${ }^{a}$ & 0.602 & $0.498-0.728$ & $<0.0001$ \\
\hline
\end{tabular}

${ }^{a}$ These variables violated the Cox model proportional hazard assumption 
Table 4

Multicovariate Cox regression model for overall survival, stratified by performance status, hormone receptor status, number of sites of metastasis, locoregional surgery, and hormonal therapy

\begin{tabular}{lccc}
\hline Parameter & Hazard ratio & $\mathbf{9 5} \%$ CI & $P$ value \\
\hline IBC versus non-IBC & 1.431 & $1.155-1.774$ & 0.0011 \\
Ethnicity & & & \\
Black versus White & 1.423 & $1.182-1.713$ & 0.0002 \\
Other versus White & 1.209 & $0.973-1.501$ & 0.0861 \\
Age at diagnosis & 1.008 & $1.001-1.014$ & 0.0207 \\
HER2 positive versus negative & 0.659 & $0.553-0.786$ & $<0.0001$ \\
Visceral versus nonvisceral metastasis & 1.206 & $1.007-1.443$ & 0.0413 \\
\hline
\end{tabular}

